University of Wollongong

Research Online

Faculty of Engineering and Information

Faculty of Engineering and Information

Sciences - Papers: Part A

Sciences

$1-1-2015$

\title{
The effect of geometry and material properties on the performance of a small hydraulic McKibben muscle system
}

\author{
Danial Sangian \\ University of Wollongong, ds706@uowmail.edu.au \\ Sina Naficy \\ University of Wollongong, snaficy@uow.edu.au \\ Geoffrey M. Spinks \\ University of Wollongong, gspinks@uow.edu.au \\ Bertrand Tondu \\ University of Toulouse
}

Follow this and additional works at: https://ro.uow.edu.au/eispapers

Part of the Engineering Commons, and the Science and Technology Studies Commons

\footnotetext{
Research Online is the open access institutional repository for the University of Wollongong. For further information
} contact the UOW Library: research-pubs@uow.edu.au 


\title{
The effect of geometry and material properties on the performance of a small hydraulic McKibben muscle system
}

\begin{abstract}
Fluidic McKibben artificial muscles are one of the most popular biomimetic actuators, showing similar static and dynamic performance to skeletal muscles. In particular, their pneumatic version offers highgenerated force, high speed and high strain in comparison to other actuators. This paper investigates the development of a small-size, fully enclosed, hydraulic McKibben muscle powered by a low voltage pump. Hydraulic McKibben muscles with an outside diameter of $6 \mathrm{~mm}$ and a length ranging from $35 \mathrm{~mm}$ to 80 $\mathrm{mm}$ were investigated. These muscles are able to generate forces up to $26 \mathrm{~N}$, strains up to $23 \%$, power to mass of $30 \mathrm{~W} / \mathrm{kg}$ and tension intensity of $1.78 \mathrm{~N} / \mathrm{mm}^{2}$ at supply water pressure of $2.5 \mathrm{bar}$. The effects of injected pressure and inner tube stiffness on the actuation strain and force generation were studied and a simple model introduced to quantitatively estimate force and stroke generated for a given input pressure. This unique actuation system is lightweight and can be easily modified to be employed in small robotic systems where large movements in short time are required.
\end{abstract}

\section{Keywords}

geometry, material, properties, performance, small, effect, hydraulic, system, mckibben, muscle

\section{Disciplines}

Engineering | Science and Technology Studies

\section{Publication Details}

Sangian, D., Naficy, S., Spinks, G. M. \& Tondu, B. (2015). The effect of geometry and material properties on the performance of a small hydraulic McKibben muscle system. Sensors and Actuators, A: Physical, 234 150-157. 


\title{
The effect of geometry and material properties on the performance of a small hydraulic
} McKibben muscle system.

\author{
Danial Sangian ${ }^{1}$, Sina Naficy ${ }^{1}$, Geoffrey M. Spinks ${ }^{1,2} *$ and Bertrand Tondu ${ }^{3}$
}

1. Intelligent Polymer Research Institute, ARC Centre of Excellence for Electromaterials Science, AIIM Facility, University of Wollongong, Wollongong, NSW 2522, Australia

2. School of Mechanical, Materials and Mechatronic Engineering, University of Wollongong, Wollongong, NSW 2522, Australia

3. Institut National de Sciences Appliquées, University of Toulouse, CNRS, 7 Avenue du Colonel Roche, 31077 Toulouse, France

* Corresponding author: E-mail: gspinks@uow.edu.au

\section{Highlights}

- A small hydraulic McKibben muscle with high efficiency is proposed.

- Effect of the bladder stiffness on Muscle performance is studied.

- A new and more accurate equation to predict the muscle performances is proposed.

- A sealed actuation system suitable for robotic machines with low voltage water pump is introduced.

\begin{abstract}
Fluidic McKibben artificial muscles are one of the most popular biomimetic actuators, showing similar static and dynamic performance to skeletal muscles. In particular, their pneumatic version offers high-generated force, high speed and high strain in comparison to other actuators. This paper investigates the development of a small-size, fully enclosed, hydraulic McKibben muscle powered by a low voltage pump. Hydraulic McKibben muscles with an outside diameter of $6 \mathrm{~mm}$ and a length ranging from $35 \mathrm{~mm}$ to $80 \mathrm{~mm}$ were investigated. These muscles are able to generate forces up to $26 \mathrm{~N}$, strains up to $23 \%$, power to mass of $30 \mathrm{~W} / \mathrm{kg}$ and tension intensity of $1.78 \mathrm{~N} / \mathrm{mm}^{2}$ at supply water pressure of 2.5 Bar. The effects of injected pressure and inner tube stiffness on the actuation strain and force generation were studied and a simple model introduced to quantitatively estimate force and stroke generated for a given input pressure. This unique actuation system is lightweight and can be easily modified to be employed in small robotic systems where large movements in short time are required.
\end{abstract}

Keywords: Actuators; Mechanical Properties; Modelling 


\section{Introduction:}

The thought of creating artificial muscles [1,2] mainly comes from two important applications. Firstly, the need for assisting human movement in cases of injury or disability [3] and secondly, for specific applications necessitating a human-like softness, such as miniaturized soft tools for small surgeries [4] and soft arms for operating in cooperation with human people [5,6]. The first stage in creation and development of an artificial muscle system is to recognize the principal engineering properties of biological muscles such as force generation, response time, actuation strain and tension intensity that need to be mimicked [2,7]. Mammalian skeletal muscles normally offer 20-40\% actuation strain and $0.35 \mathrm{~N} / \mathrm{mm}^{2}$ tension intensity in less than one second with power to mass of 100 W/kg [8]. Traditionally, large stroke actuation is achieved with piston-cylinder actuators that offer high forces. These systems are difficult to seal, are heavy and bulky, especially considering the pumps and compressors needed and they also suffer from static friction [9]. In the last two decades, a wide range of artificial muscles has been introduced and developed [7,10]. Pneumatic artificial muscles (PAMs) in particular have shown a great capability for fabricating robots and surgery tools because of their similarity to biological muscles as well as high actuation force per mass [11-15].

Joseph L. McKibben was the first to introduce McKibben artificial muscle as the most important type of PAMs in the late 1950s to assist paralyzed patients. The muscle normally includes an expandable inner tube surrounded by a braided sleeve and operated by the injection of pressurized air into the inner tube. Once the inner tube is pressurized, the volume of the inner tube increases and it expands in a balloon style against the braided sleeve. The braid constrains the expanded inner tube to maintain a cylindrical shape to amplify stroke or force along the braid axis. This particular artificial muscle however presents some disadvantages such as the requirement of a separated mechanical air compressor, a noisy system due to exhaust during depressurization, a heavy system to carry for human or robots and high electricity consumption [16]. To overcome mentioned disadvantages several attempts have recently been made to replace the air with chemo - sensitive materials to mimic how biological muscles transform chemical energy into mechanical energy. For instance, Tondu et al [17] introduced a new type of McKibben muscle by replacing air with $\mathrm{pH}$ sensitive hydrogel spheres, which offers reasonable performances but suffers from a long response time ( > $30 \mathrm{~min}$ ).

Hydraulic McKibben muscles have been introduced recently as a new generation of these muscles, which operate with pressurized water or oil [18,19]. As noted by Tiwari et al.[20] and Meller et al. [21], the use of bulky compressors can be avoided in hydraulic artificial muscles (HAMs), thus making compact design possible. Meller et al.[21] have also clearly demonstrated that the HAMs have approximately doubled the energy conversion efficiency of PAMs. Moreover, it has been 
shown that it was possible to design relatively 'large' pneumatic McKibben muscles to actuate human limb-size robots [22], or even very powerful ones thanks to the use of a particular strong external braided sleeve [23]. In an alternative way, it is also possible to develop microscale HAMs like the ones proposed by Moon, Ryu, Chun et al.[16], or by Sodano and Rotinat-Libersa [24] for millimeter scale robot development. The purpose of the work reported in this paper is to investigate small-size hydraulic McKibben muscles working in a limited pressure range as developed by Tiwari, Meller et al. [20], but with the additional constraint of functioning using small, low voltage pumps suitable for portable applications.

Here we present a cost-effective (US\$ 2), fast and small hydraulic McKibben muscle (35 - $80 \mathrm{~mm}$ resting length) that offers $23 \%$ actuation stroke (under $4.9 \mathrm{~N}$ isotonic load) and up to $26 \mathrm{~N}$ isometric force generated in about one second. The muscle simply operates with pressurized water that is supplied from a small container $(25 \mathrm{ml})$ in a fully sealed system that can be easily carried in portable applications. The electricity consumption of this particular muscle is significantly low $(6 \mathrm{~V}$, $0.14 \mathrm{~A}$ ) and operates with small batteries. We have also investigated the effect of muscle length and the stiffness of the inner tube on muscle performances such as force generation, contraction ratio and response time.

\section{Prototype design:}

\subsection{Sample fabrication:}

McKibben artificial muscles normally consist of four important parts: an elastic tube, a cylindrical reinforcement braid, and two connectors for the pressurized fluid supply. To determine the effect of inner tube stiffness on the actuator performance, inner tubes made of natural rubber latex with two different thicknesses $(0.28$ and $0.56 \mathrm{~mm})$ and a silicone rubber tube (thickness of $1 \mathrm{~mm}$, Holman Flex tube) were used inside the braids. All bladders had an external diameter of $4.5-5.0 \mathrm{~mm}$. Cylindrical braids with thickness of $0.44 \mathrm{~mm}$ and outer radius in the unstrained state $\left(\mathrm{R}_{\mathrm{o}}\right)$ of $3 \mathrm{~mm}$, made from polyphenylene sulfide (PPS) were obtained from JDD TECH Company-China. Crescent zip lock was also used to connect the muscle to tube connections. The initial angle $\left(\alpha_{0}\right)$ of the braided sleeve (Fig.1) was kept constant at $35^{\circ}$. The muscle was fabricated as follows: first, the inner tube was cut into the desired length of 35,50 , or $80 \mathrm{~mm}$. Next, the inner tube was inserted into the braided sleeve, and finally the PVC connector tubes were glued to both ends of the inner tube. 

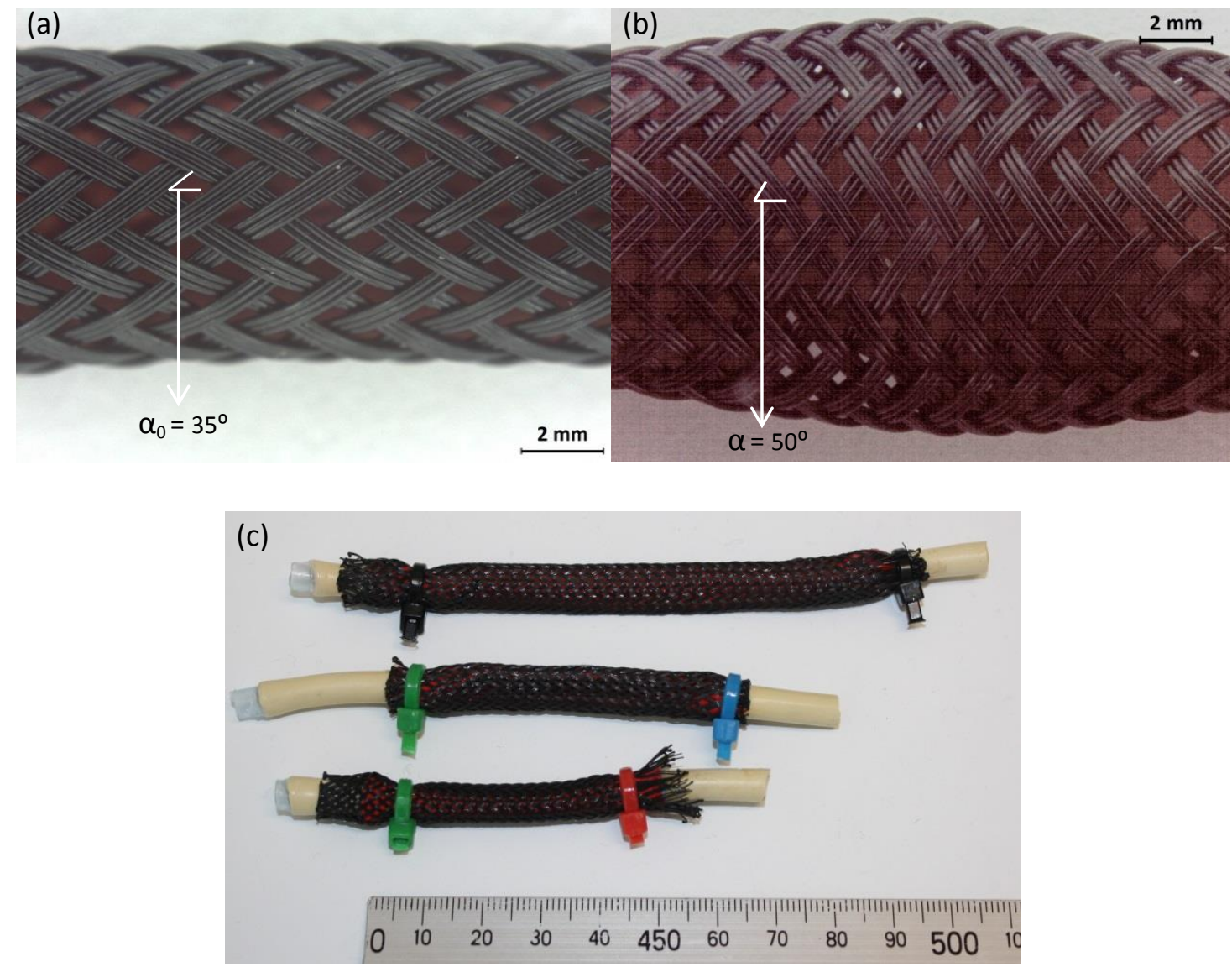

Fig.1. Polymeric braided sleeve used for hydraulic McKibben muscle. (a) resting state (b) expanded state. (c) Photographs of HAMs of different lengths with tube connectors attached.

\subsection{Test setup:}

The experimental set up was specially designed to measure actuation strain, generated force, response time and water pressure. The sealed actuation system consisted of four important parts: a low voltage water pump (6 V, flow rate: $0.5 \mathrm{~L} / \mathrm{min}$, Flodos / NF6 KPDCB), a small water container (25 ml), McKibben muscle and a manual valve. Care was taken when filling the actuator and connecting tubes with water so as to remove any trapped air. The entire system had a total weight of only $350 \mathrm{~g}$, making it easily usable in robotic machines. The main mass of the system was due to the pump and power supply which can be optimized depending on the application. The operation is simply by opening and closing the manual valve. When the valve is closed while the pump is working the water becomes pressurized inside the muscle and causing the muscle to contract. Dualmode lever system machine (Aurora Scientific, Model 300B) and Shimadzu EZ tensile machine were employed to record actuation stroke and force generated. The lever arm and tensile machine were connected to the artificial muscle, while the other end of the muscle was fixed. An e-corder data logger (ED 410, e-DAQ) was used to connect the lever arm unit to a PC, and e-DAQ Chart was 
used to record the data. The internal water pressure inside the muscle was also monitored by using a digital pressure meter. The current and voltage applied to the pump were noted periodically.

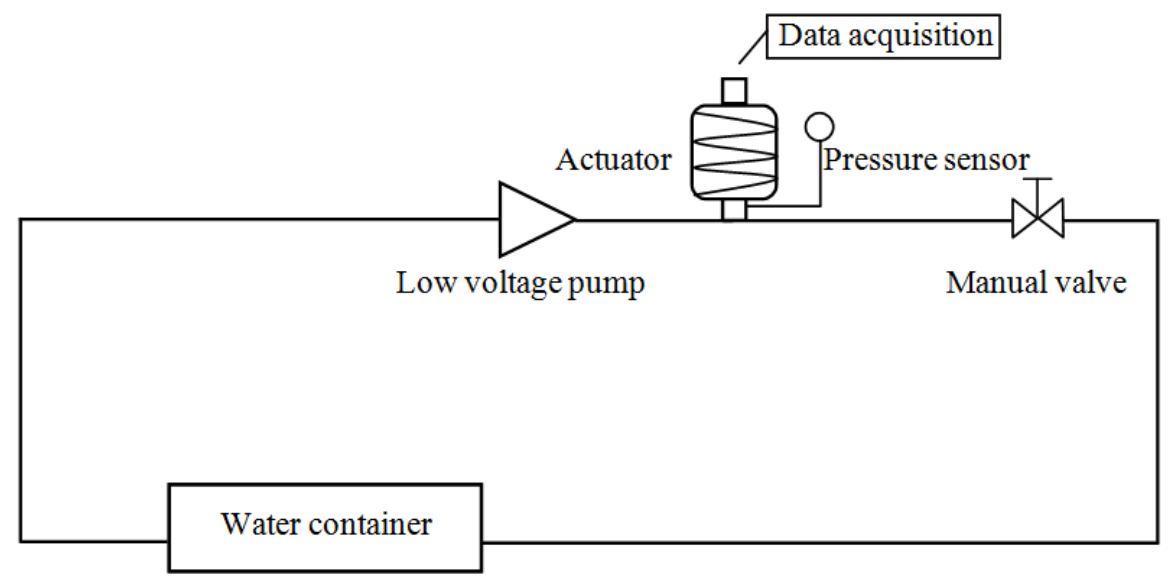

Fig.2.Schematic view of the actuation setup used for hydraulic McKibben muscle

\subsection{Experimental techniques:}

In this paper, both isometric and isotonic standard tests for pneumatic artificial muscles were performed to obtain actuation results in accordance with corresponding physiological definitions:

- Isometric contraction: The muscle length was fixed to be constantly equal to its initial length and the maximum muscle force generation (the 'blocked force') recorded by using a force sensor.

- Isotonic contraction: A given tensile force of $4.9 \mathrm{~N}$ was applied to the muscle by using a lever arm. This method was used to record the isotonic length variation of the muscle.

- Force-stroke curves: The possible force / stroke combinations were obtained by first measuring the isometric blocked force and then allowing the muscle to contract while simultaneously measuring force and stroke.

In all of these tests the pressure supplied by the water pump was kept constant.

\section{Results and Discussion:}

\subsection{Effect of the unloaded muscle length on isometric force generation and isotonic actuation strain} with constant pressure:

An analysis of actuation length effects on the response time and isotonic strain behaviors was made by comparing three different muscles lengths of 35, 50 and $80 \mathrm{~mm}$ under load of $4.9 \mathrm{~N}$ and water pressure of 2.5 bar $(250 \mathrm{kPa}$ ) applied for $\sim 1$ second and then released. Figure. 3 (a) exhibits that all muscles contracted continuously during the pressurization period with the shortest length $(35 \mathrm{~mm})$ achieving a strain of $23 \%$ in about 1 second, while the muscle with lengths of 50 and $80 \mathrm{~mm}$ generated smaller actuation strains of 18.5 and $16 \%$, respectively. The time to reach a target strain 
of $15 \%$ increased with starting muscle length from 0.5 seconds $(35 \mathrm{~mm})$ to 0.7 seconds (50 $\mathrm{mm}$ ) and 0.9 seconds $(80 \mathrm{~mm})$. The faster response seen in the shorter muscle was likely attributed to the smaller volume of water needed to pressurize the muscle. The expansion response time due to depressurizing the muscles was also dependent on muscle length but considerably faster than pressurization in all cases. Furthermore, the muscle of $80 \mathrm{~mm}$ produces the highest actuation displacement and greatest power (0.075 Watts) compared to the other two muscles with shorter lengths. The power was calculated during contraction as the product of displacement and load per contraction time and is shown in Figure $3 \mathrm{~b}$. The overall power conversion efficiency was $8.9 \%$ based on the input electrical power of $0.84 \mathrm{~W}$ needed for the hydraulic pump.

Isometric force generation is one of the essential requirements for many applications of artificial muscles such as robotic surgery and artificial jumping legs. According to Volder et al [14] high forces of $1-10 \mathrm{~N}$ are required for robotic devices and surgery tools. Here we compared the three different HAM muscles with water pressure of 2.5 bar to investigate the effect of actuation length on muscle performance. The maximum isometric force of $26 \mathrm{~N}$ was recorded for the $80 \mathrm{~mm}$ long muscle in just 1.4 seconds. According to Figure 3, the muscle ability in force generation scales approximately with actuator length. The shortest muscle $(35 \mathrm{~mm})$ generates isometric force of $11 \mathrm{~N}$ in 1.2 seconds, which is almost half that of the muscle with $80 \mathrm{~mm}$ length. These results were very consistent for five consecutive pressurization - depressurization tests. Previous studies have shown little effect of braid length on the force generated when pressurized [25]. However, our muscles have comparatively small aspect ratios and below the recommended ratio of 14 [26] so that end effects may limit the force generated.

The performances of our muscles are compared with previous HAMs systems, as summarized in Table 1. The reported systems vary considerably in size and operating pressures. The reported maximum (blocked) forces covered a wide range with the larger diameter muscles generated the higher forces. Three previous studies used similarly small diameter braids as used in the present work of less than $6 \mathrm{~mm}[16,20,24]$. The maximum contraction (free) strains from these small diameter braids were of a similar magnitude ( 20\%) and the maximum blocked forces were either similar or lower than those reported in the present study. The comparison highlights that it is possible to generate HAM performance comparable with other literature studies with the use of a low voltage / low power electric pump and a limited pressure range of 2.5 bar. 
Table 1. Performance comparison of HAMs reported in the literature and the present study. Maximum values are shown for stroke and blocked force and minimum values for response time. Values in parentheses for this work indicate the HAM muscle length used in the present study.

\begin{tabular}{|c|c|c|c|c|c|c|}
\hline $\begin{array}{c}\text { Muscle } \\
\text { Performances }\end{array}$ & $\begin{array}{l}\text { Tiwari et } \\
\text { al. [20] }\end{array}$ & $\begin{array}{c}\text { Mori et al. } \\
{[23]}\end{array}$ & $\begin{array}{l}\text { Moon et al. } \\
{[16]}\end{array}$ & $\begin{array}{l}\text { Solano et } \\
\text { al. [24] }\end{array}$ & $\begin{array}{l}\text { Meller et } \\
\text { al. [21] }\end{array}$ & This work \\
\hline $\begin{array}{l}\text { Muscle length } \\
(\mathrm{mm})\end{array}$ & 160 & 700 & 237 & 61 & 173 & $\begin{array}{l}35 \\
50 \\
80\end{array}$ \\
\hline Diameter(mm) & 3.17 & 40 & 2 & 1.5 & 32.3 & 6 \\
\hline Braid angle $\left({ }^{\circ}\right)$ & ---- & 15 & ---- & 17 & 28.7 & 35 \\
\hline $\begin{array}{l}\text { Muscle weight } \\
\text { (kg) }\end{array}$ & 0.00182 & 2.96 & 0.23 & 0.02 & --- & 0.0018 \\
\hline Pump type & --- & $\begin{array}{l}\text { Water hand } \\
\text { pump }\end{array}$ & $\begin{array}{l}9 \mathrm{~V} \text { electric } \\
\text { pump }\end{array}$ & $\begin{array}{l}\text { Motor and } \\
\text { piston }\end{array}$ & ---- & $\begin{array}{l}6 \mathrm{~V} \text { electric } \\
\text { pump }\end{array}$ \\
\hline Pressure(bar) & 5.5 & 40 & 6 & 10 & 7 & 2.5 \\
\hline $\begin{array}{l}\text { Free stroke } \\
\text { (\%) }\end{array}$ & 19 & 25 & 11 & $\begin{array}{c}21 \\
\text { (under } 200 \mathrm{gr} \\
\text { constant } \\
\text { weight) }\end{array}$ & $\begin{array}{c}30 \\
\text { (LDPE bladder) }\end{array}$ & $\begin{array}{l}21(35) \\
23(50) \\
28(80)\end{array}$ \\
\hline $\begin{array}{l}\text { Blocked force } \\
\text { (N) }\end{array}$ & 23 & 28000 & 3.8 & 6 & $\begin{array}{c}500 \\
\text { (LDPE bladder) }\end{array}$ & $\begin{array}{l}15(35) \\
22(50) \\
26(80)\end{array}$ \\
\hline $\begin{array}{l}\text { Response time } \\
\text { (sec) }\end{array}$ & --- & ---- & 2.8 & ---- & --- & 1.1 \\
\hline
\end{tabular}

(a)

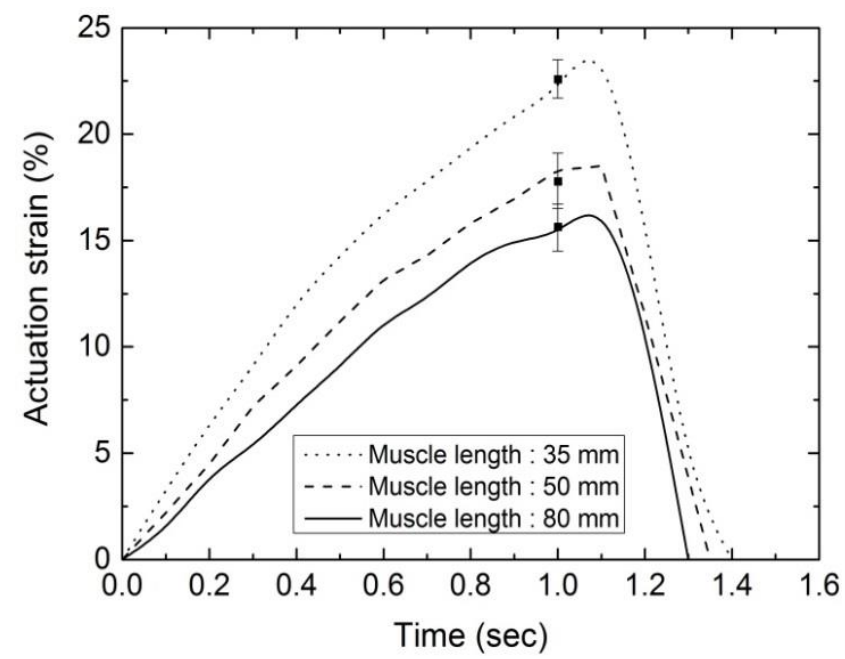

(b)

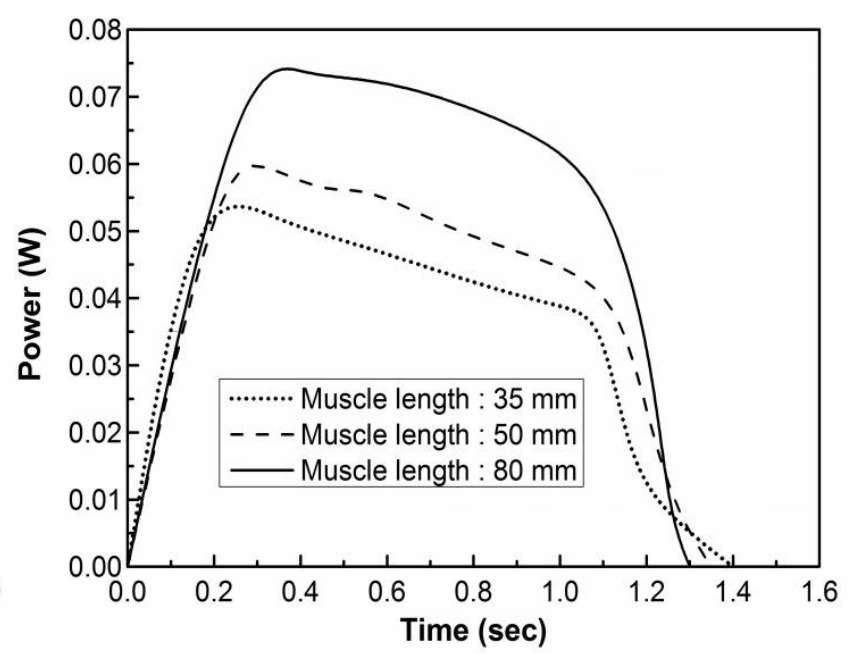




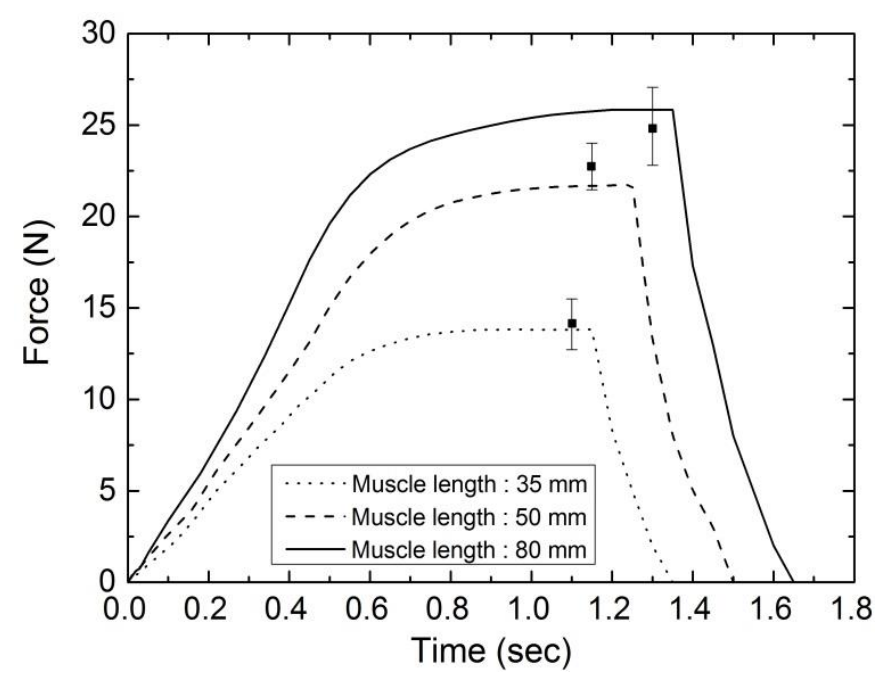

Fig. 3. Dynamic behavior of the HAM muscle (a) Isotonic actuation test under constant water pressure (2.5 bar) and given load of $4.9 \mathrm{~N}$; (b) Corresponding power output from isotonic test; (c) Isometric force test under constant water pressure (2.5 bar). Pressurization - depressurization tests were performed four times on each HAM and the average maximum strains and forces for each HAM length are shown by the solid squares in (a) and (c). The ranges of maximum strain and force values are represented by the error bars calculated as one standard deviation around the mean.

\subsection{Effect of the inner tube stiffness on HAM performances:}

A classical model of an ideal purely cylindrical McKibben artificial muscle relates the static force $F$ produced by the muscle to its control pressure $P$ and its contraction strain $\varepsilon$ [27]. This model can take the following form:

$$
F(P, \varepsilon)=\left(\pi R_{0}^{2}\right) P\left[a(1-\varepsilon)^{2}-b\right], \quad 0 \leq \varepsilon \leq \varepsilon_{\max }
$$

Where $R_{0}$ is the initial radius of braid, $a=3 / \tan ^{2} \alpha_{0}, b=1 / \sin ^{2} \alpha_{0}$. The model predicts that the maximum contraction strain is independent of applied pressure and is given by:

$$
\varepsilon_{\max }=1-\frac{1}{\sqrt{3} \cos \alpha_{o}}
$$

This model only requires the knowledge of two geometric parameters characterizing the artificial muscle: the initial braid angle $\alpha_{0}$ and the initial muscle radius $r_{0}$, which is usually assumed to be the initial external inner tube radius, and considered as being equal to the initial internal braided sleeve radius. This model assumes a full transmission of the pressurized stress inside the inner rubber tube 
to the external braided sleeve. Such an assumption is generally verified in the case of pneumatic artificial muscles working in a typical [1bar-5bars]-range if a sufficiently thin inner tube made of a soft rubber was chosen. Meller et al.[21] have recently demonstrated the effects of bladder stiffness on HAM performance with increasingly stiff bladders significantly limiting the maximum strain achieved and slightly reducing the blocked force. A semi-empirical approach was introduced to account for bladder stiffness in the ideal model by introducing fitting parameters $\kappa_{F}$ and $\kappa_{\varepsilon}$ for force and strain, respectively [21]:

$$
F=\kappa_{F}\left(\pi r_{o}^{2}\right) P\left[a\left(1-\kappa_{\varepsilon} \varepsilon\right)^{2}-b\right]
$$

where, $\kappa_{F}$ is the ratio of the measured blocked force to the maximum force predicted by equation (1) and $\kappa_{\varepsilon}$ is the ratio of the maximum strain predicted by equation (1) to the measured maximum strain.

Here we also consider the effect of bladder stiffness on HAM performance and introduce an alternative approach to modelling. First experiments focused on the effect of bladder and braid stiffness on the pressure needed to develop an isometric stress in the range of $1-4 \mathrm{~N}$ and the corresponding free stroke. Three different individual muscles with the same geometry (35mm long and $6 \mathrm{~mm}$ diameter braid) were made with three different inner tube stiffnesses of 78, 150 and 490 $\mathrm{N} / \mathrm{m}$. Stiffnesses were measured as the slope of force versus extension curves obtained by axial stretching of the inner tube bladders. The data of Figure 4 shows that the muscle with the stiffest inner tube needs dramatically more water pressure $(2.14$ bar) to reach the targeted static force of 1.2 $\mathrm{N}$ and exhibits just $2.5 \%$ contraction free strain. In contrast, the muscle with smallest inner tube stiffness $(78 \mathrm{~N} / \mathrm{m})$ needs only 0.33 bar pressure for the same amount of force and shows $4 \%$ contraction strains (Fig 4.a). All tested samples showed a similar degree of hysteresis in the loading and unloading curves that has been attributed previously to braid friction [27].

(a)

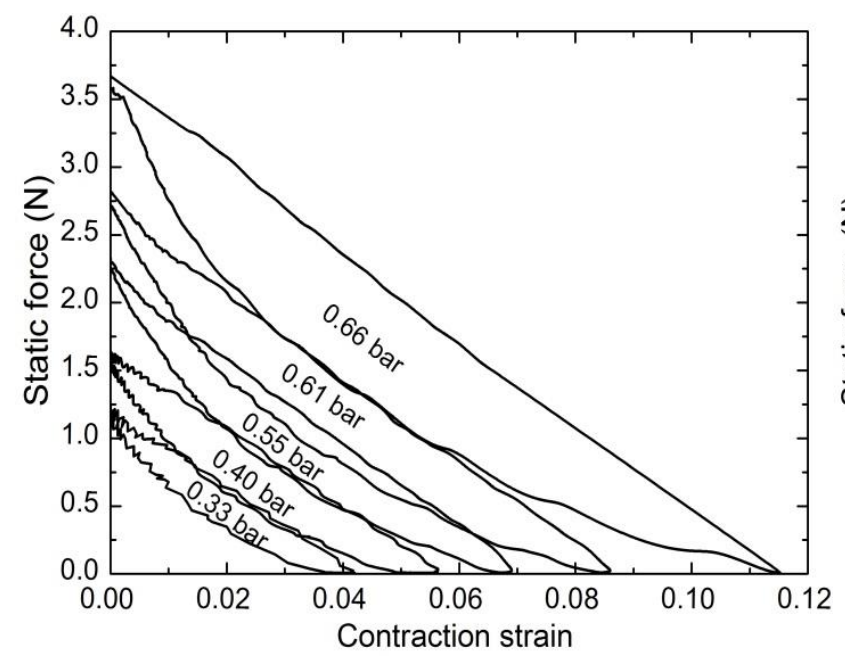

(b)

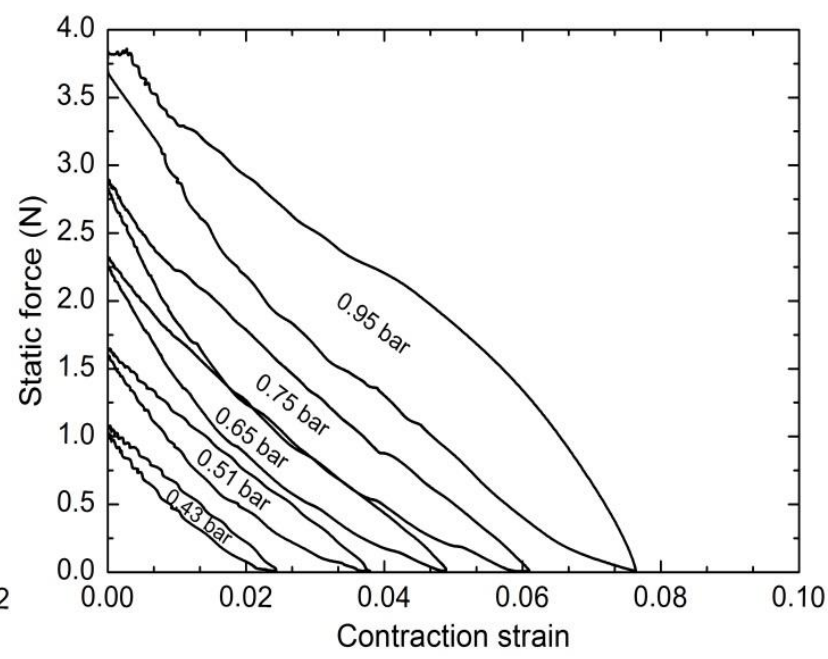


(c)

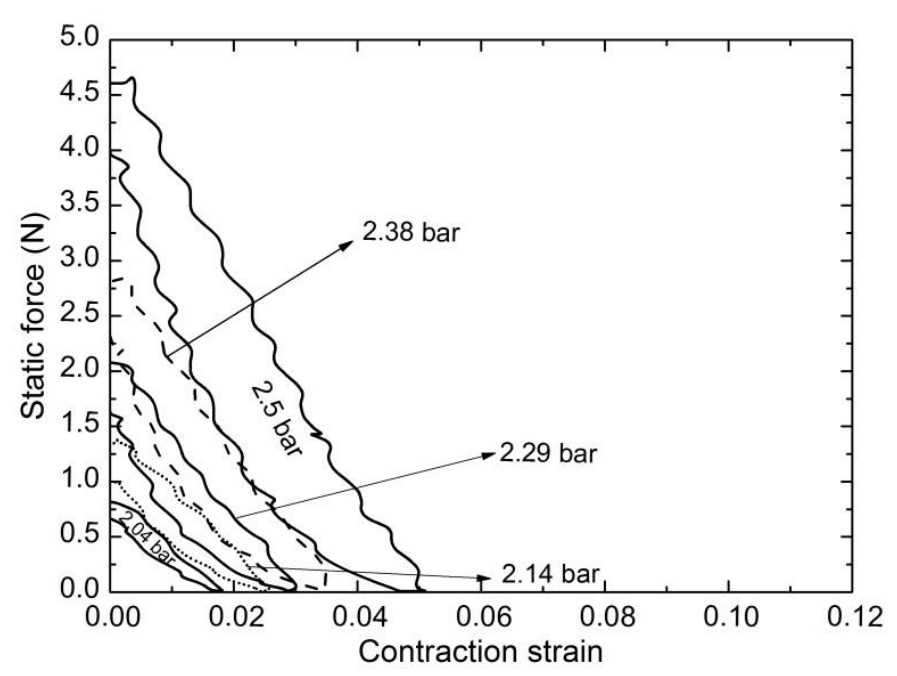

Fig.4. Typical static forces and contraction strains produced by a hydraulic artificial muscle emphasizing the role of input pressure and illustrating the hysteresis phenomenon and the fundamental role of the stiffness of the bladder. The muscles with initial length of $35 \mathrm{~mm}$ and inner tube stiffness of (a) $78 \mathrm{~N} / \mathrm{m}$ (b) $150 \mathrm{~N} / \mathrm{m}$ and (c) $490 \mathrm{~N} / \mathrm{m}$. Note the noise seen in Figure 4(c) is from pump vibration that is more prominent at the high pressures used for this sample.

The above results confirm that HAM performance is influenced by parameters other than the initial braid angle and radius, as suggested by equation (1). All contraction strains were less than $12 \%$ which is well below the prediction of $30 \%$ from Equation (2) based on a starting braid fiber angle of $35^{\circ}$. Blocked forces for a given input pressure were also considerably lower than the predicted values. Increasing bladder stiffness tended to decrease the blocked force and contraction strain, perhaps as a result of the elastic deformation of the bladder and friction between the bladder and braid.

A second set of experiments was performed to further investigate the effect of bladder stiffness on HAM performance. Longer samples lengths of $80 \mathrm{~mm}$ were used to reduce end-effects that diminish achievable contraction strains. Three different bladders were used and the results shown in Figure 5 demonstrate that contraction ratios now approach the predicted value of $30 \%$, at least at the higher input pressures. For example, the HAM using the least stiff bladder and pressurized to 2.5 bar gave a maximum contraction ratio of $28 \%$. However, even with these longer samples, it is clear that the increasing bladder stiffness reduces both the blocked force and contraction ratio for a given input pressure. 
(a)

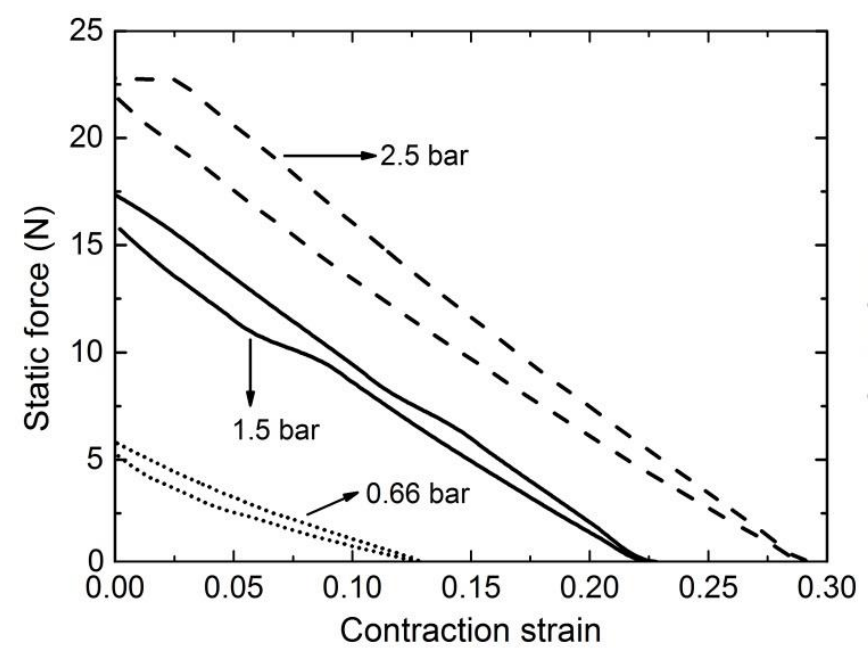

(b)

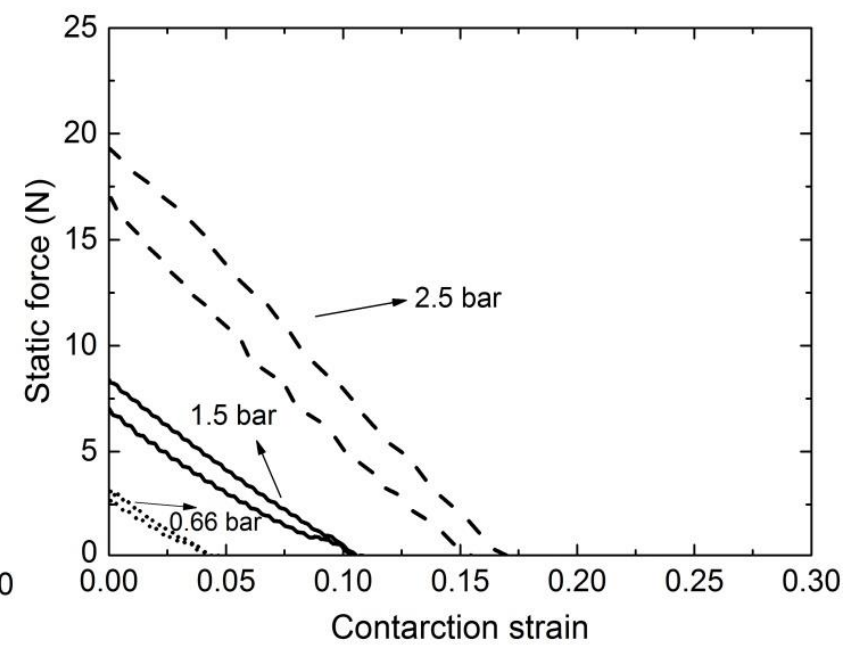

(c)

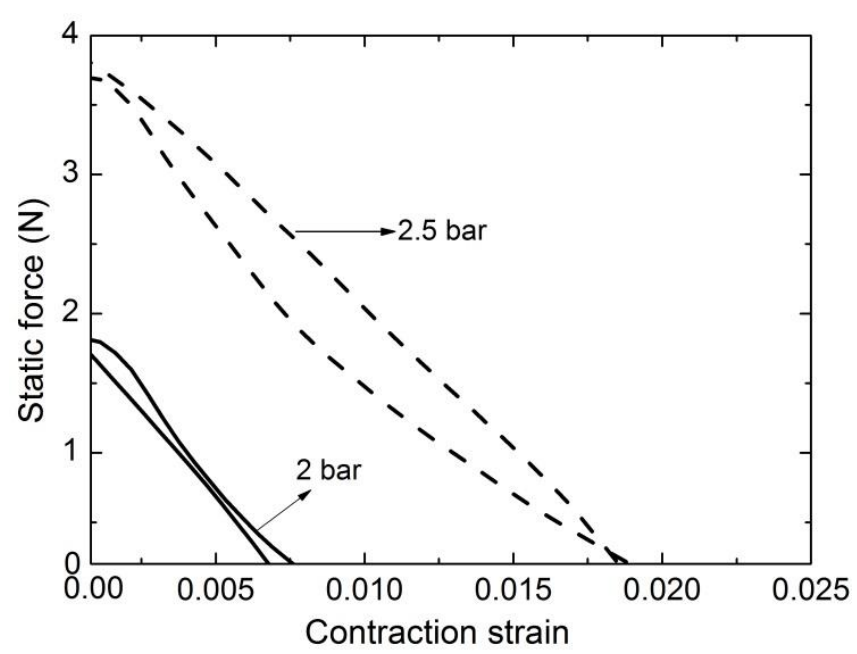

Fig.5. Typical static forces and contraction strains produced by hydraulic artificial muscles at different input pressures. The muscles with initial length of $80 \mathrm{~mm}$ and inner tube stiffness of (a) $78 \mathrm{~N} / \mathrm{m}$ (b) $150 \mathrm{~N} / \mathrm{m}$ (c) 490 $\mathrm{N} / \mathrm{m}$.

In an attempt to quantify the effect of the bladder stiffness on HAM performance, we consider that the elastic resistance due to the inflation of the bladder material tends to reduce the pressure available and restricts the McKibben muscle output. Here we offer a simple approach to account for the pressure needed to inflate the bladder. We use the ideal McKibben muscle relation of Equation (1) but modify the input pressure by subtracting two contributions: the threshold pressure needed to inflate the bladder to make contact with the braid $\left(P_{t h}\right)$; and the pressure needed to elastically deform the bladder when in contact with the braid and causing HAM contraction. The threshold pressure is a fixed term that depends on the bladder and braid geometries and the bladder stiffness and occurs when the bladder outer diameter is smaller than the braid inner diameter. Figure 6 shows 
the measured blocked force at several input pressures for the three different bladders used in the present study. Also shown is the theoretical blocked force obtained from Equation (1). These results show that the variation in blocked force with pressure are similar for all bladders and correspond quite closely to the expected trend. However, the measured results are offset along the pressure axis by an amount that increases with bladder stiffness. Extrapolating the experimentally measured values for each bladder material to zero force provides an experimental measure of the threshold pressure, $P_{t h}$. These values are given in Table 2. For pressures below the threshold the bladder is not yet in contact with the braid and no force is generated by the muscle.

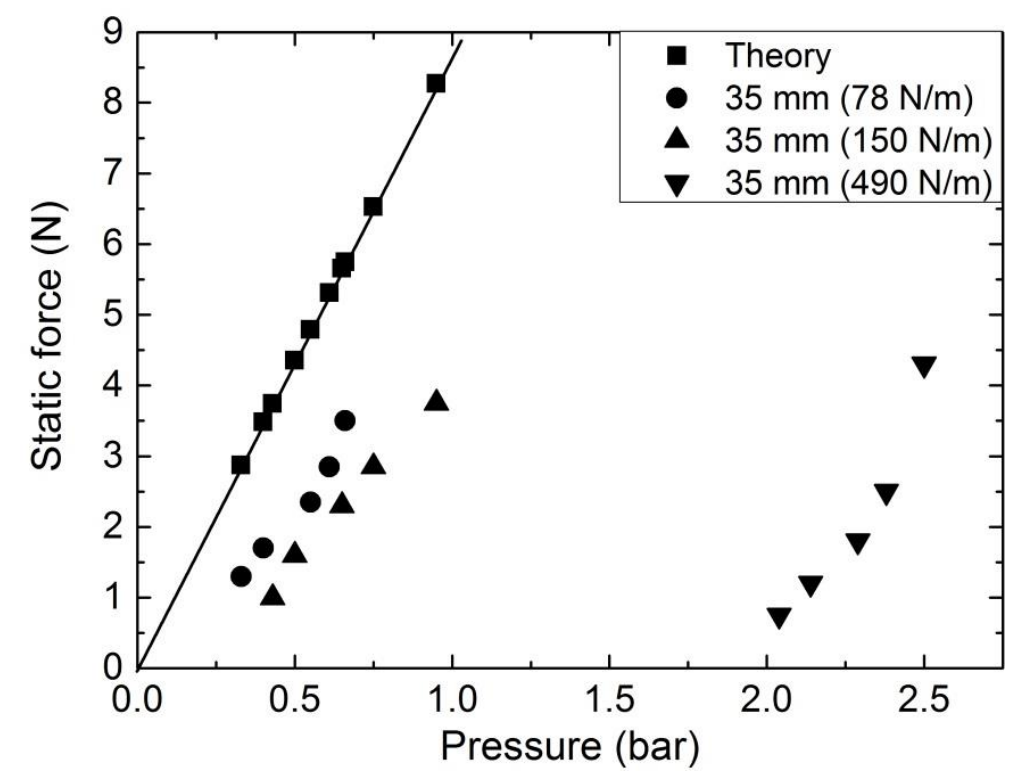

Fig.6. Static (blocked) forces measured at various input pressures for HAMs made with 3 different bladder materials: circular and triangular symbols are measured values. The linear line with square symbols is the theoretical values obtained from Equation (1) that assumes an ideally thin bladder. 
Table 2. Threshold pressure and parameters for each bladder.

\begin{tabular}{|lccc|}
\hline $\begin{array}{l}\text { Physical Information of } \\
\text { bladder materials }\end{array}$ & $\mathbf{7 8 ~ N / m}$ & $\mathbf{1 5 0 ~ N / m}$ & $\mathbf{4 9 0} \mathbf{~ N} / \mathbf{m}$ \\
Threshold Pressure $\mathrm{P}_{\mathrm{th}}$ (bar) & 0.08 & 0.21 & 1.89 \\
Elastic Modulus (MPa) & 0.9 & 0.9 & 0.8 \\
Inner Diameter (mm) & 5.1 & 4.5 & 3.0 \\
Outer Diameter (mm) & 5.6 & 5.6 & 5.0 \\
Wall Thickness (mm) & 0.28 & 0.56 & 1.0 \\
\hline
\end{tabular}

The measured input pressure was also adjusted by subtracting the pressure needed to elastically deform the bladder $\left(P_{e l}\right)$ when in contact with the braid during HAM contraction. This pressure was estimated for simplicity by the standard mechanics analysis of a pressurized cylinder where the circumferential strain in the bladder is given by:

$$
\varepsilon_{b}=\frac{\Delta r}{r_{o}}=\frac{P r_{o}}{E_{b} t_{b}}
$$

Where $P$ is the internal pressure; $\Delta r=r-r_{o}$ with $r$ and $r_{o}$ representing the pressurized and unpressurized inner radius of the bladder; and $E_{b}$ and $t_{b}$ are the bladder elastic modulus and wall thickness, respectively. When $P$ reaches the threshold pressure $\left(P=P_{t h}\right)$, the bladder makes first contact with the braid. We assume that for pressures in excess of the threshold $\left(P>P_{t h}\right)$, the bladder is in contact with the braid so that outer radius of the bladder $\left(r+t_{b}\right)$ is the same as the inner radius of the braid $(R)$. The change in braid radius is then estimated by:

$$
\Delta R=\frac{r_{o}^{2}}{E_{b} t_{b}}\left(P_{e l}-P_{t h}\right)
$$

where $P_{e l}$ is the pressure needed to further inflate the bladder so that it maintains contact with the braid during HAM contraction. The change in braid length can be determined using the geometry relations appropriate for the helically wound fibers in the braid:

$$
R=\frac{b \sin \alpha}{2 \pi N} \quad \text { and } \quad L=b \cos \alpha
$$

Where the braid of length $L$ is constructed from inextensible fibers of length $b$ and wrapped $N$ times at an angle of $\alpha$ to the braid long axis. Combining these equations gives the following relation that shows that the pressure needed to elastically expand the bladder increases with increasing HAM contraction strain. 


$$
P_{e l}=P_{t h}+\frac{E_{b} t_{b} b}{2 \pi N r_{o}^{2}}\left[\left(1-\frac{L^{2}}{L_{o}^{2}} \cos ^{2} \alpha_{o}\right)^{1 / 2}-\sin \alpha_{o}\right]
$$

where $L_{o}$ and $\alpha_{o}$ are the starting braid length and fiber wrap angle.

Table 2 lists the geometry and elastic modulus of each bladder material and these values are used to estimate the expected contraction strain, as shown in Figure 7. The approach is to use the ideal McKibben muscle relation of Equation (1) modified as:

$$
F(P, \varepsilon)=\left(\pi R_{0}^{2}\right) P^{*}\left[a(1-\varepsilon)^{2}-b\right], \quad 0 \leq \varepsilon \leq \varepsilon_{\max }
$$

With the input pressure $P^{*}=P_{\text {applied }}-P_{e l}$ where $P_{\text {applied }}$ is the actual applied pressure that is modified by subtracting the pressure needed to elastically deform the bladder $\left(P_{e l}\right)$ calculated from equation (7).

As shown in Figure 7, the calculated values from this modified model are in reasonable agreement with the measured values. In contrast, the standard McKibben muscle relation of equation (1) does not include the effect of bladder stiffness and, as shown by the dotted lines in Fig. 7, this standard model over-estimates both the force and strain generated when stiffer bladders were used. The subtraction of the threshold pressure in the modified model ensures that the calculated blocked maximum force is in closer agreement with the measured values, since the unmodified pressure greatly over-estimates the achievable force when stiffer bladders were used. The subtraction of the pressure needed to elastically deform the bladder during HAM contraction can also significantly reduce the maximum contraction strain. The theoretical prediction using unmodified pressure predicts the same maximum strain regardless of the bladder material used or input pressure. The measured values clearly show that the maximum strain increases with increasing input pressure and is affected by the bladder stiffness. These effects are reasonably modelled using the simple approach applied here. While the modified model captures the main trends, there remain discrepancies between the calculated and measured force / strain values. The modified model is based on material linear elasticity and assumes a constant bladder wall thickness. Both assumptions are not strictly valid for the large strains occurring in the elastomeric bladders used with the HAMs. However, incorporating non-linear elasticity effects adds significant complexity to the model and was not considered in the present study. 

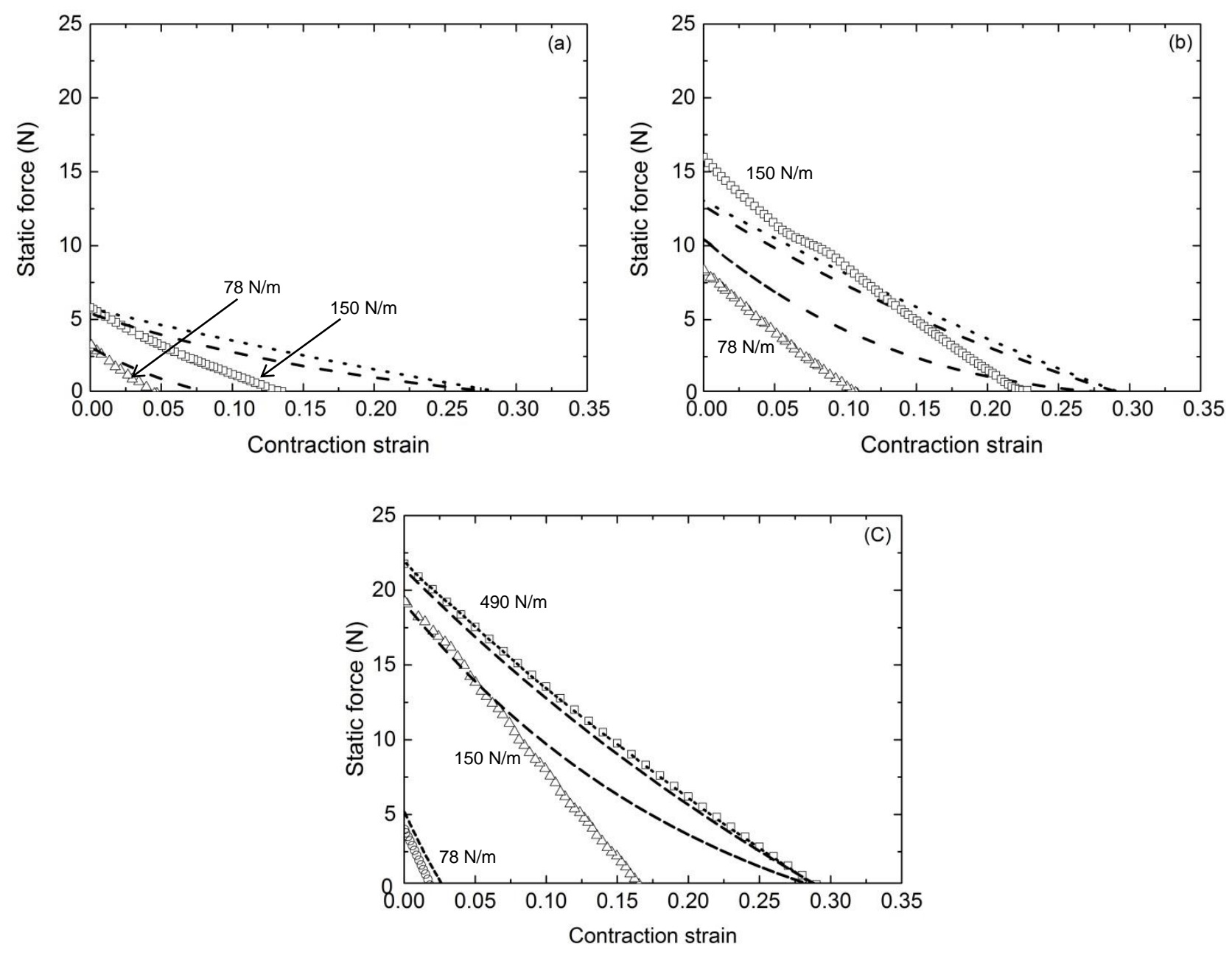

Fig.7. Comparison of measured and calculated force-strain curves for $80 \mathrm{~mm}$ long HAM pressurized to a) 0.66 bar; b) 1.5 bar and c) 2.5 bar. Calculated values using the standard McKibben muscle relationship (equation 1) are shown by the dotted lines. Experimentally measured values and values calculated using the modified model (equations 7 and 8 ) are shown by the symbols and dashed lines, respectively, where the squares are for the $78 \mathrm{~N} / \mathrm{m}$ bladder; triangles represent the $150 \mathrm{~N} / \mathrm{m}$ bladder; and circles are for the $490 \mathrm{~N} / \mathrm{m}$ bladder (part c only).

\section{Conclusions:}

Hydraulic McKibben artificial muscles are easy to manufacture, and perform quite similar to biological muscles in terms of response time, isotonic actuation strain and isometric force generation. We scaled these actuators down to a diameter of $6 \mathrm{~mm}$ and lengths of $35-80 \mathrm{~mm}$, which makes them more suitable to perform in applications such as robotic fingers for surgical tools. Forces up to $26 \mathrm{~N}$ were achieved at a pressure of $2.5 \mathrm{bar}$, with an overall system response time of just 1.4 seconds. Actuation strain of $23 \%$ was obtained in just 1.1 seconds with given load of $0.5 \mathrm{~kg}$ and 2.5 bar. It has been found that the effect of stiffness of the inner tube on muscle performances is considerable and should be carefully chosen. In particular, the pressure needed to inflate the bladder 
to make contact with the braid reduces the maximum force achievable. Secondly, the pressure needed to elastically deform the braid during HAM contraction reduces the maximum achievable strain. A simple method was proposed to quantitatively estimate these effects with reasonable accuracy. The best performing HAM muscles at low pressures generated by low-voltage portable water pumps were achieved with the least stiff bladders. Further work will consider the cycle life implications of using such thin bladders.

\section{Acknowledgments}

The authors acknowledge the Australian Research Council for financial support of this work through its Professorial Fellowship scheme (DP110101073).

\section{References:}

[1] R.H. Baughman, Conducting polymer artificial muscles, Synthetic Metals 78 (1996) 339-353.

[2] J.D. Madden, Materials science - Artificial muscle begins to breathe, Science 311 (2006) 15591560 .

[3] K.E. Gordon, G.S. Sawicki, D.P. Ferris, Mechanical performance of artificial pneumatic muscles to power an ankle-foot orthosis, J Biomech 39 (2006) 1832-1841.

[4] D. Tarnita, D.N. Tarnita, N. Bizdoaca, I. Mindrila, M. Vasilescu, Properties and medical applications of shape memory alloys, Romanian Journal of Morphology and Embryology 50 (2009) $15-21$.

[5] D. Villegas, M. Van Damme, B. Vanderborght, P. Beyl, D. Lefeber, Third-Generation Pleated Pneumatic Artificial Muscles for Robotic Applications: Development and Comparison with McKibben Muscle, Advanced Robotics 26 (2010) 1205-1227.

[6] P. Tommasino, D. Caligiore, V. Sperati, G. Baldassarre, A McKibben Muscle Arm Learning Equilibrium Postures, 4th Ieee Ras \& Embs International Conference on Biomedical Robotics and Biomechatronics (2012), 1229-1234.

[7] J.D. Madden, N.A. Vandesteeg, P.A. Anquetil, P.G.A. Madden, A. Takshi, R.Z. Pytel, S.R. Lafontaine, P.A. Wieringa, I.W. Hunter, Artificial muscle technology: physical principles and naval prospects. Oceanic Engineering, IEEE Journal of 29 (2004) 706-728.

[8] I.W. Hunter, S. Lafontaine, A Comparison of Muscle with Artificial Actuators, Solid-State and Actuators Workshop 5th Technical Digest IEEE (1992) 178-185.

[9] J.F. Blackburn, Fluid Power Control, MIT Press, Cambridge, 1960.

[10] T. Mirfakhrai, J.D.W. Madden, R.H. Baughman, Polymer artificial muscles, Materials Today 10 (2007) 30-38.

[11] B. Tondu, P. Lopez, The McKibben muscle and its use in actuating robot-arms showing similarities with human arm behaviour, Ind Robot 24 (1997) 432-439.

[12] R.W. Colbrunn, G.M. Nelson, R.D. Quinn, Modeling of braided pneumatic actuators for robotic control, International Conference on Intelligent Robots and Systems 4 (2001) 1964-1970.

[13] Y.K. Lee, I. Shimoyama, A multi-channel micro valve for micro pneumatic artificial muscle, Proc Ieee Micr Elect (2002) 702-705.

[14] M. De Volder, A.J.M. Moers, D. Reynaerts, Fabrication and control of miniature McKibben actuators, Sensors and Actuators A: Physical 166 (2011) 111-116.

[15] Z.Y. Zhang, M. Philen, Pressurized artificial muscles, J Intel Mat Syst Str 23 (2012) 255-268. 
[16] K.W. Moon, D. Ryu, C. Chun, Y. Lee, S. Kang, M. Park, Development of a slim haptic glove using McKibben artificial muscles 2006 SICE-ICASE International Joint Conference 1-13 (2006) 3942-3946.

[17] B. Tondu, S. Mathe, R. Emirkhanian, Low pH-range control of McKibben polymeric artificial muscles, Sensor Actuat a-Phys 159 (2010) 73-78.

[18] K.K.K. Ku, R.S. Bradbeer, K.K.Y. Lam, L.F. Yeung, R.C.W. Li, A Novel Actuator for Underwater Robots, IEEE Journal of Oceanic Engineering 34 (2009) 331-342.

[19] M. De Volder, D. Reynaerts, Pneumatic and hydraulic microactuators: a review, J Micromech Microeng 20 (2010) 1-18.

[20] R. Tiwari, M.A. Meller, K.B. Wajcs, C. Moses, I. Reveles, .E Garcia, Hydraulic Artificial Muscles, J Intel Mat Syst Str 23(2012) 301-312.

[21] M.A. Meller, M. Bryant, E. Garcia, Reconsidering the McKibben muscle: Energetics, operating fluid, and bladder material, Journal of Intelligent Material Systems and structures (2014)

1-18, DOI: $10.1177 / 1045389 \times 14549872$.

[22] R.M. Robinson, C.S. Kothera, B.K.S. Woods, High Specific Power Actuators for Robotic Manipulators, Journal of Intelligent Material Systems and Structures 22 (2011) 1501-1511.

[23] M. Mori, K. Suzumori, M. Takahashi, T. Hosoya, Very High Force Hydraulic McKibben Artificial Muscle with a p-Phenylene-2,6-benzobisoxazole Cord Sleeve, Advanced Robotics 24 (2010) 233-254.

[24] B. Solano, C. Rotinat-Libersa, Compact and Light-weight Hydraulic Actuation System for High Performance Millimeter Scale Robotic Applications: Modeling and Experiments, Journal of Intelligent Material Systems and Structures 22 (2011) 1479-1487.

[25] C.S. Kothera, M. Jangid, J. Sirohi and N.M. Wereley, Experimental characterization and static modeling of McKibben Actuators, J. Mech.Design, 131 (2009) 091010.

[26] G.K. Klute, B. Hannaford, Accounting for elastic energy storage in McKibben artificial muscle actuators, ASME J. Dynamic Syst, Meas.Control, 122 (2000) 386-388.

[27] B. Tondu, Modelling of the McKibben artificial muscle: A review. J Intel Mat Syst Str 23(2012) 225-253. 